\title{
Transcriptomic analysis of genes in soybean in response to Peronospora manshurica infection
}

Hang Dong ${ }^{1,2 \dagger}$, Shuangfeng Shi ${ }^{1 \dagger}$, Chong Zhang ${ }^{2 \dagger}$, Sihui Zhu ${ }^{1,2}$, Mei Li ${ }^{1}$, Jie Tan', Yue Yu ${ }^{3}$, Liping Lin', Shirong Jia', Xujing Wang ${ }^{1}$, Yuanhua $\mathrm{Wu}^{2^{*}}$ and Yuhui Liu ${ }^{1 *}$ (D)

\begin{abstract}
Background: Soybean downy mildew (SDM), caused by Peronospora manshurica (Pm), is a major fungal disease in soybean. To date, little is known regarding the defense mechanism at molecular level and how soybean plants response to Pm infection. In this study, differential gene expression in SDM-resistant (HR) and SDM-susceptible (HS) genotype was analyzed by RNA-seq to identify differentially expressed genes (DEGs) following Pm infection.

Results: Of a total of 55,017 genes mapped to the soybean reference genome sequences, 2581 DEGs were identified. Clustering analysis of DEGs revealed that these genes could be grouped into 8 clusters with distinct expression patterns. Functional annotation based on gene ontology (GO) and KEGG analysis indicated they involved in diverse metabolism pathways. Of particular interest were the detected DEGs participating in SA/ROS and JA signalling transduction and plant/pathogen interaction.

Conclusion: Totally, 52 DEGs with $P$ value $<0.001$ and $\log _{2}$ fold change $>2$ or $<-2$ upon fungal inoculation were identified, suggesting they were SDM defense responsive genes. These findings have paved way in further functional characterization of candidate genes and subsequently can be used in breeding of elite soybean varieties with better SDM-resistance.
\end{abstract}

Keywords: Soybean downy mildew, RNA-seq analysis, Differentially expressed genes, Transcription factors, Disease resistance, Soybean

\section{Background}

Soybean (Glycine max L.) is a major crop worldwide that provides abundant amount of protein and oil for human and animal consumption. However, soybean was severely affected by an obnoxious fungal disease, downy mildew (SDM), caused by Peronospora manshurica (Pm). The yield loss could be as high as $6-15 \%$ on average in the years when it was epidemic [1-4]. The proliferation of this fungus was favored by high humidity and relatively low temperature at $20-22{ }^{\circ} \mathrm{C}$. Initially infected leaves had pale green to light yellow spots on the upper surface

\footnotetext{
* Correspondence: wuyh7799@163.com; liuyuhui@caas.cn

${ }^{\dagger}$ Equal contributors

${ }^{2}$ College of Plant Protection, Shenyang Agricultural University, Shenyang, China

${ }^{1}$ Biotechnology Research Institute, Chinese Academy of Agricultural Sciences, Beijing, China

Full list of author information is available at the end of the article
}

but later on these spots were creamy white to pinkish coupled with growth of fruiting bodies on the back side of leaves leading to chlorophyll degradation and premature defoliation. In addition, the fungi obtain nutrients exclusively from living plant cells and cannot be cultured apart from their host $[5,6]$.

Although some fungicides can be applied to reduce SDM infection, the most effective way to control this disease and reduce yield loss is to develop and use resistant variety in soybean production. In our previous study [7], 15 soybean genotypes were either in vitro diseaseindexed via leaf/ $P m$ inoculation or by field observation in two consecutive years. By which the genotypes were classified into five categories: HR (highly resistant), $\mathrm{R}$ (resistant), MR (moderate resistant), S (susceptible) and HS (highly susceptible). We had cloned a GmSAGT1 gene coding for a bi-functional enzyme of serine/alanine

(c) The Author(s). 2018 Open Access This article is distributed under the terms of the Creative Commons Attribution 4.0 International License (http://creativecommons.org/licenses/by/4.0/), which permits unrestricted use, distribution, and reproduction in any medium, provided you give appropriate credit to the original author(s) and the source, provide a link to the Creative Commons license, and indicate if changes were made. The Creative Commons Public Domain Dedication waiver (http://creativecommons.org/publicdomain/zero/1.0/) applies to the data made available in this article, unless otherwise stated. 
glyoxylate aminotransferase from a soybean genotype highly resistant to SDM. Fifteen soybean genotypes were used for qRT-PCR analysis, which showed the average expression level of GmSAGT1 in HR group was 13 times of that in HS group. Northern blot analysis indicated that the expression level of GmSAGT1 in HS, S, MR and $\mathrm{R}$ group was 5, 22, 39 and $65 \%$ of that in HR, respectively. Transgenic tobacco expressing GmSAGT1 increased resistance to a fungal pathogen Rhizoctonia solani, demonstrating that higher expression of GmSAGT1 gene was closely correlated with increased SDM resistance.

However, the genomic mechanism associated with SDM resistance is currently not known that needs to be further elucidated. Understanding the genetic regulation of plant defense system will help to identify specific genes for either gene-based marker selection or genetic engineering to develop soybeans with better disease resistance. With the advent of next-generation sequencing technology, Illumina sequencing approach has been used for understanding the complexity of gene expression and regulation networks in plants responding to different pathogen attack, including bacterial leaf pustule (Xanthomonas axonopodis pv. glycines) [8, 9], Phytophthora root and stem rot (Phytophthora sojae) [10], Asian soybean rust (Phakopsora pachyrhizi) [11] in soybean, and soybean cyst nematode (SCN; Heterodera glycines) in common bean [12]. By which the responsive genes and metabolism pathways associated with disease resistance have been identified. Unfortunately, no such a study on soybean/Pm interaction has yet been reported so far.

To gain transcriptional profiling data after $\mathrm{Pm}$ infection, a highly SDM-resistant genotype (HR) Jilinxiaoli1 (JL1) and a highly susceptible (HS) genotype Kefeng1
(KF1) were used for comparison in this study. The differentially expressed genes of HR and HS at $72 \mathrm{~h}$ after inoculation (hai) was compared with that obtained from non-inoculated leaves. Totally, 52 DEGs with $P$ value $<0.001$ and $\log _{2}$ fold change $>2$ or $<-2$ upon fungal inoculation were identified. Of particular interest was the detected DEGs might involve in SA (salicylic acid), ROS (reactive oxygen species) and jasmonic acid (JA) signaling, implicating their roles in response to $P m$ invasion. A high level of accumulation of defense responsive gene products such as pathogenesis-related (PR) proteins and NBS-LRR (nucleotide binding site-leucine rich repeat) might also contribute to SDM-resistance in soybean. In addition, some transcription factors (TFs) of bHLHs, MYBs and WRKYs were up-regulated after inoculation. These findings provided an insight for further functional characterization of candidate genes resistant to SDM that would help in improving soybean breeding program. To the best of our knowledge, this is the first report describing differential expression of genes in response to soybean $/ \mathrm{Pm}$ interaction.

\section{Results \\ SDM symptoms}

Symptoms on leaves of JL1 (HR) and KF1 (HS) were investigated 72 hai. There were a large number of brown lesions surrounded by yellow haloes on the surface of leaves of HS KF1. In contrast, only a few number of small yellow spots on the leaves of HR JL1 formed (Fig. 1). The behavior of HR and HS genotype after inoculation was as expected, indicating their leaf samples could be used for further transcriptomic analysis.
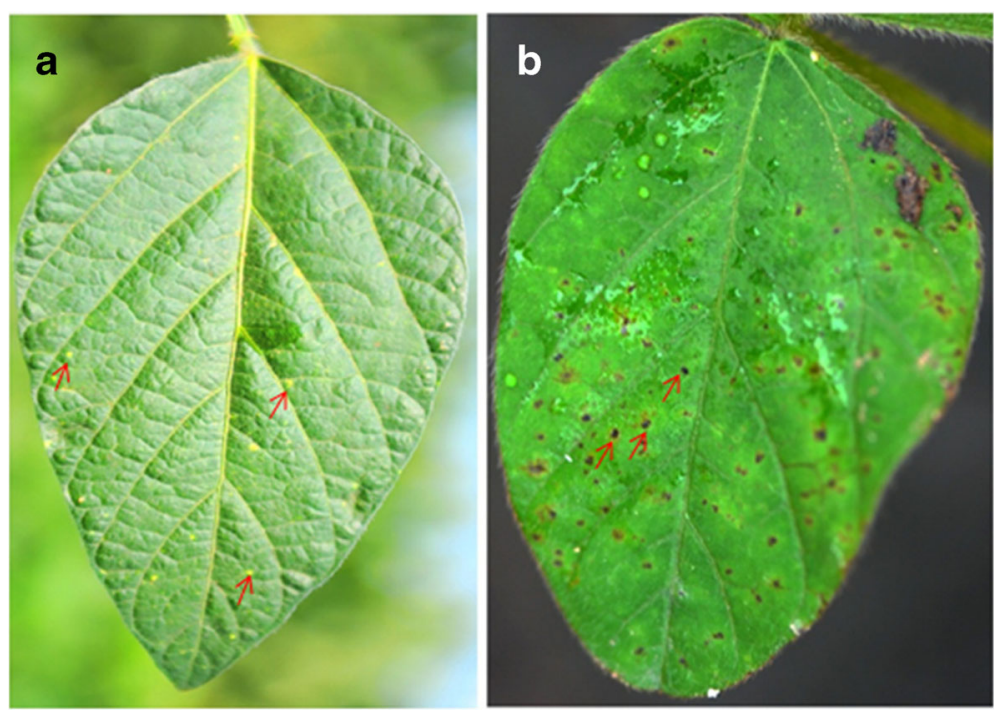

Fig. 1 SDM-symptoms on leaves of HR/JL1 and HS/KF1. a \& b: showing SDM-symptoms on leaves of JL1 and KF1 $72 \mathrm{~h}$ after inoculation (hai) with fungal pathogen $P$. manshurica 


\section{RNA-seq analysis}

Changes in transcript levels between inoculated and non-inoculated (JL1i vs. JL1ni and KF1i vs. KF1ni) were analyzed by RNA-seq. A total of 128.22 million raw reads were generated by 75 bp single end sequencing from the four cDNA libraries, constituting $4.51 \mathrm{~Gb}$ of cDNA sequences per library on average (Additional file 1: Table S1). By trimming adaptor sequences and elimination of low quality reads as well as reads containing unknown nucleotides larger than $10 \%$, a total of 120.07 million clean reads were generated. Of which, 97.00 and 95.75 million clean reads were mapped (80.78\%) and uniquely mapped $(79.47 \%)$, respectively, to the soybean genome reference sequence (Glycine $\max$ Wm82.a2.v1) by using TopHat software (Additional file 2: Table S2). Of 65,781 predicted genes in the soybean genome, the expression level of 55,017 mapped genes were quantified based on RPKM values (reads per kilo bases per million reads) converted from mapped read counts of DEGs.

\section{Transcriptome analysis in response to $\mathrm{Pm}$-inoculation}

Using the random sampling model in the DEGseq program, the mapped read counts of each gene with a $P$ value $<0.001$ and $\log _{2}$ fold change $>2.0$ or $<-2.0$ were selected, by which a total of 2581 DEGs were obtained.

In comparison of gene expression level in the noninoculated samples (JL1ni vs. KF1ni), the expression level of 1174 and 937 DEGs were higher or lower in JL1 than that in KF1 (Fig. 2a), respectively, representing genotypic differences between HR and HS genotype. In the inoculated samples, the expression level of 164 genes were
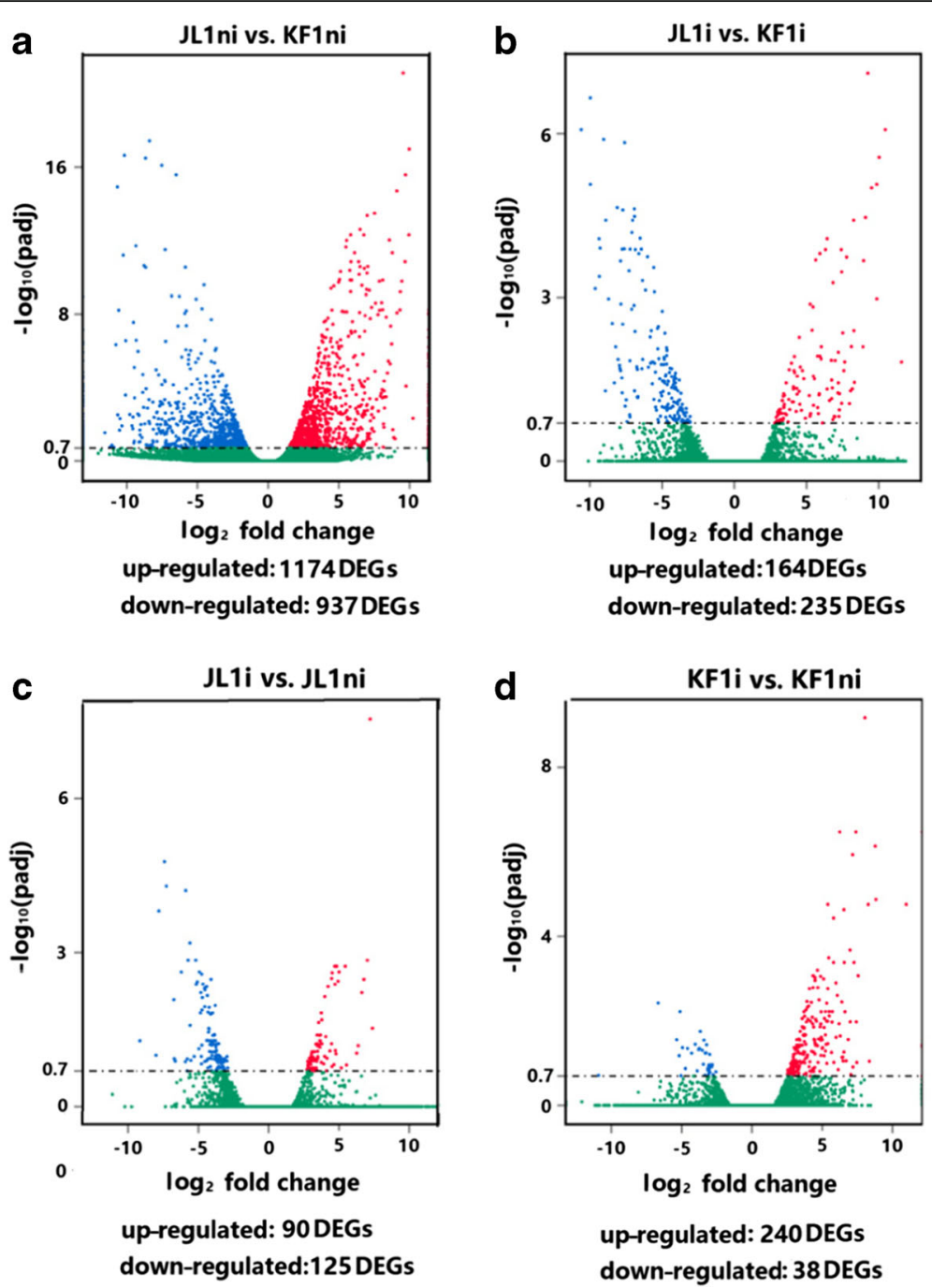

Fig. 2 Transcriptional expression of genes in inoculated and non-inoculated leaves of HR/JL1 and HS/KF1. a: displaying the log fold change in expression of genes in JL1ni vs. KF1ni). b: differentially expressed genes (DEGs) in JL1i vs. KF1i). c: DEGs in JL1i vs. JL1ni. d: DEGs in KF1i vs. KF1ni. Y-axes indicates the mean of normalized counts (padj <0.05) and X-axes indicates the $\log _{2}$ fold change values. DEGs are shown in blue, green and red indicating down-regulated, no change and up-regulated genes, respectively 
higher and 235 genes were lower in JL1 than that in KF1, implying these genes might be specifically involved in the process of soybean $/ \mathrm{Pm}$ interaction (Fig. $2 \mathrm{~b}$ ).

In terms of paired comparison of up- and downregulated DEGs in the inoculated vs. non-inoculated of HR or HS genotype, it was found that in the HR/JL1, out of 215 DEGs 90 were up-regulated and 125 were down-regulated (Figs. 2c and 3), while in the HS/KF1, of a total of 278 DEGs, 240 genes were up-regulated and 38 down-regulated, respectively (Figs. $2 \mathrm{~d}$ and 3 ).

\section{Clustering of DEGs}

$\mathrm{H}$-clustering analysis was used to group 2581 DEGs into clusters and sub-clusters based on common expression patterns. Eight distinct sub-clusters $\mathrm{A}$ to $\mathrm{H}$ were generated reflecting the general trends and key transitional states in the HR and HS following Pm-inoculation. The number of genes within each cluster was presented in Fig. 4.

\section{Gene ontology (GO)}

Based on the PFAM domain (http://pfam.xfam.org/), the 2581 DEGs were converted into GO-identities by mapping (http://www.geneontology.org). Mapped GO was then classified into three categories: biological process, cellular component and molecular function (Additional file 3: Table S3).

To understand the functional components involved in molecular responses to $P m$, we annotated the most enriched GO terms in the HR/JL1 and HS/KF1 with

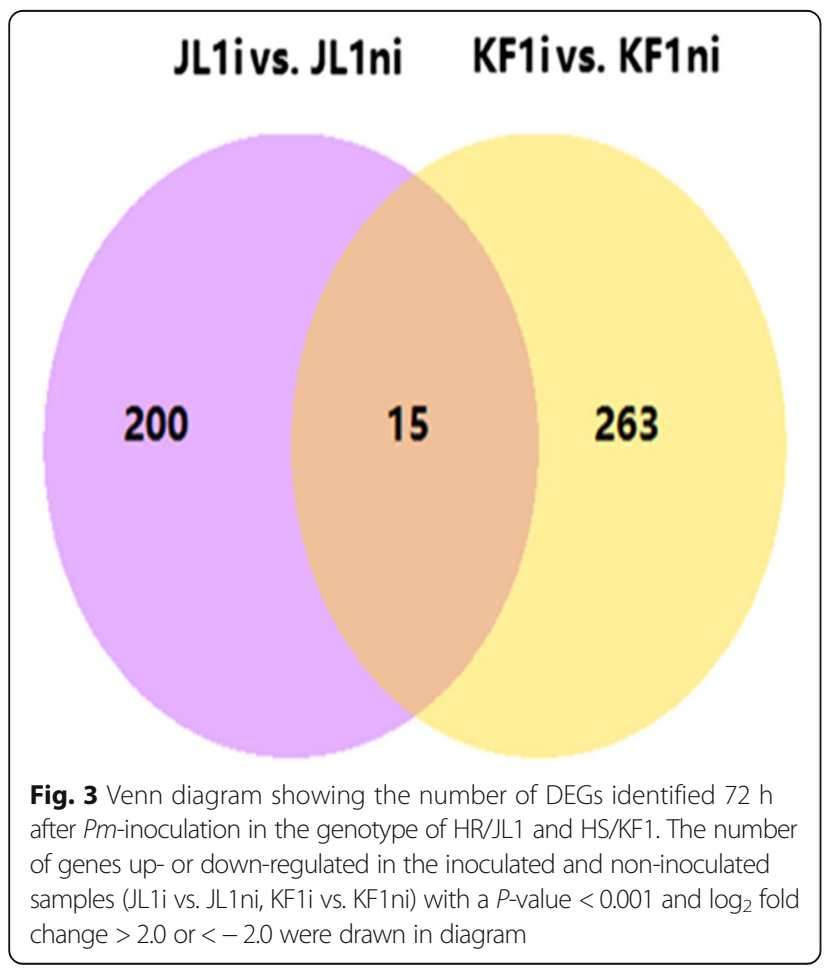

significant difference at a level of corrected $P$ value $<0.05$ (Table 1).

It was notable that the DEGs related to photosystem were all down-regulated upon Pm-inoculation in both HR and HS genotypes. Under the category of biological process, 7 DEGs associated with photosynthesis and 2 DEGs related to thyazole metabolic process were downregulated in the HR genotype. Similarly, in the category of cellular component, 7 enriched DEGs related to photosynthesis and 7 DEGs associated with thylakoid were down-regulated in HR genotype, while in the HS genotype 4 DEGs in the category of biological process and 4 DEGs in the cellular component were also downregulated. This common feature reflects inhibition of photosystem in soybean plants upon Pm-infection.

In contrast to the reduction of photosynthesis, 5 of the most enriched DEGs under the category of biological process and 4 DEGs in the category of molecular function, that all associated with ion homeostasis (e.g. oxidizing metal ions, ferric iron binding, ferroxidase and oxidoreductase activity), were up-regulated in HR genotype, implicating modification of diverse ion channels after inoculation might play a vital role in defense responses to $P m$ invasion (Table 1).

\section{KEGG pathway analysis}

In doing KEGG analysis, 2581 DEGs were annotated to KEGG pathway (http://www.genome.jp/kegg/), by which a total of 216 DEGs were identified. Results showed that in HR/JL1, among a total of 107 DEGs, there were 64 DEGs up-regulated involving in 31 KEGG pathways and 43 DEGs down-regulated participating in 19 different KEGG pathways (Additional file 4: Table S4). In HS/ KF1, of 109 DEGs, 91 were up-regulated involving in 26 KEGG pathways and 18 were down-regulated involving in 13 KEGG pathways (Additional file 5: Table S5).

Apart from the basic metabolic pathways, e.g. carbohydrate, amino acid and lipid metabolism, the most interest to us were pathways participating phytohormone signalling transduction and plant/pathogen interactions based on the knowledge already published in the literature.

In the plant hormone signaling, two genes encoding either EFB1/2 (EIN3-binding F-box protein1-like, involving in ethylene signaling) or AUX/IAA protein (auxinresponsive protein IAA, involving in auxin signaling) were identified in JL1i vs. JL1ni (Table 2), while in KF1i vs. KF1ni two AUX/IAA genes and seven JAZ (JASMONATE ZIM-motif) genes (involving in jasmonate signaling), and one BAK1 protein kinase gene (Brassinosteroid insensitive1-associated receptor kinase1) were detected (Table 2). In addition, in the glyoxylate and dicarboxylate metabolism, two genes coding for isocitrate lyase, a key catalytic enzyme involving in ROS signaling, were identified. All of these results demonstrated that these genes 

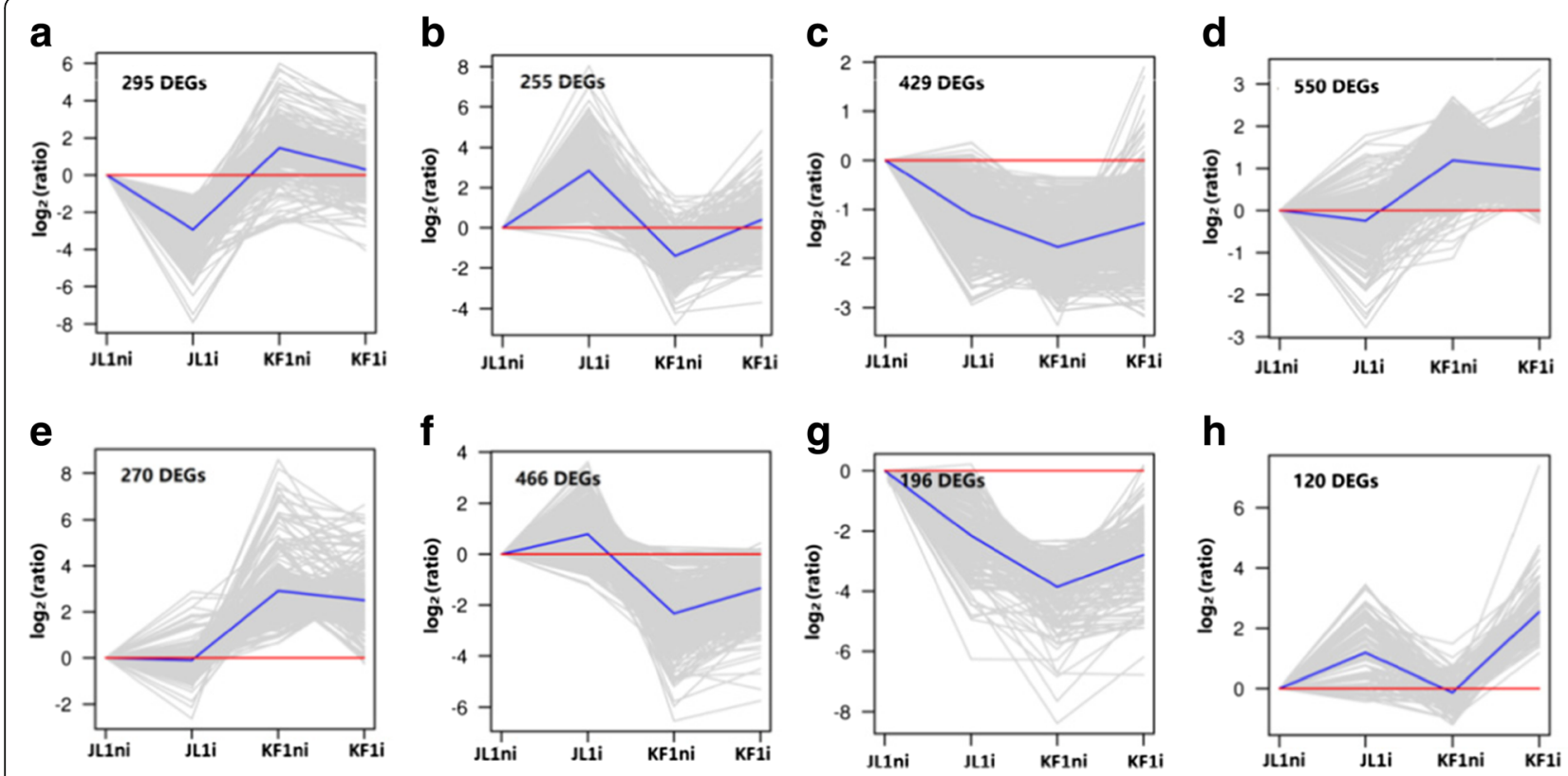

Fig. 4 Clustering of 2581 detected DEGs. Genes were classified into eight subclusters $\mathbf{a}$-h based on similarity of expression pattern. The gray lines represent the relative expression level of each gene within each sub-cluster from 4 libraries of JL1i, JL1ni, KF1i and KF1ni. The blue lines represent the mean of the relative expression level of all genes within each sub-cluster

were actively involving in the complex phytohormone signaling pathways.

In the plant pathogen interaction, three genes in JL1i vs. JL1ni were detected including genes coding for LRR receptor-like serine/threonine-protein kinase FLS2 protein (Flagellin-sensitive 2), calcium-binding protein CML, and a protein associated with cyclic nucleotide gated channel (Table 2). In KFi vs. KF1ni, totally 18 genes were identified, including seven JAZ genes, seven calmodulin genes, one BAK1, one DNA-binding WRKY (Glyma01g31921), and one FLS2 (Table 2).

Except one calmodulin gene was down-regulated, all above mentioned DEGs in the pathways of phytohormone signal transduction and plant/pathogen interaction were up-regulated in both genotypes of HR and HS after inoculation with $\mathrm{Pm}$ (Table 2).

\section{SDM defense responsive genes}

In searching SDM defense responsive genes, a total of 52 DEGs either up- or down-regulated were identified. Of the total, 31, 11 and 10 DEGs were categorized into phytohormone signalling transduction, plant/pathogen interaction and TFs, respectively (Table 2).

In the phytohormone signalling pathway, $12,8,7,1,1$ and 2 DEGs participating calmodulin, SA/ROS, JA, ET, BR (brassinosteroid) and AUX (auxin), respectively, in response to $P m$-infection. In particular, 3 and 4 peroxidase genes were highly expressed with 4-6-fold increase in HR/JL1 and 7-9-fold increase in HS/KF1 by comparison of inoculated with non-inoculated samples. In the category of plant/pathogen interaction, $11 \mathrm{PR}$ proteins were involved. In terms of TFs, 1 bHLH, 6 MYBs and 3 WRKYs were identified in the study (Table 2).

By comparison of the expression level of the 52 DEGs in non-inoculated samples of JL1ni and KF1ni, if the read counts of KFni was taken as 1 , then the $\log _{2}$ fold change of 38 DEGs in JL1ni were higher than that in KF1ni, accounting for $73 \%$ of the total DEGs. Noteworthy, there were 14 DEGs associated with plant/pathogen interaction (including $5 \mathrm{PR}$ proteins, 5 calmodulins and 4 oxidative proteins) and 3 transcription factors, all of which with $\log _{2}$ fold change value greater than 2.0 , demonstrating that the basal expression level of these DEGs were much higher in HR genotype without fungal pathogen and might be served as basal defense responsive genes (Table 2).

On the other hand, when comparing the data of HR and HS after fungal inoculation, it was found that 50 DEGs in KF1i were up-regulated with $\log _{2}$ fold change all greater than 2,0, accounting for $96 \%$ of the total. While in the JL1i, 43 DEGs (83\%) with $\log _{2}$ fold change greater than 2,0 were found after pathogen attack. These data suggested that the HS responded to Pm infection more quickly and strongly. By counting the number of DEGs, however, it was found that the read counts of 30 DEGs in JL1i were still higher than that in KF1i (Table 2).

\section{qRT-PCR validation}

To validate the RNA-seq expression data and its reliability, seven DEGs were randomly selected for Real-time quantitative PCR (qRT-PCR) analysis. To compare these 
Table 1 The most enriched GO terms in HR/JL1 and HS/KF1 with significant difference at a level of corrected $P$ value $<0.05$

\begin{tabular}{|c|c|c|}
\hline GO terms & GO ID & Gene ID \\
\hline \multicolumn{3}{|l|}{ JL1i vs. JL1ni Up-regulated } \\
\hline \multicolumn{3}{|l|}{ Biological process } \\
\hline Iron ion homeostasis & GO:0006879, GO:0055072, GO:0006826 & \multirow{5}{*}{$\begin{array}{l}\text { Glyma01g31300, Glyma03g06420, Glyma07g19060, } \\
\text { Glyma18g43650 }\end{array}$} \\
\hline Metal ion homeostasis & $\begin{array}{l}\text { GO:0046916, GO:0055076, GO:0006875, GO:0055065, } \\
\text { GO:0000041 }\end{array}$ & \\
\hline Cation homeostasis & GO:0030003, GO:0055080 & \\
\hline Ion homeostasis & GO:0006873, GO:0050801, GO:0098771 & \\
\hline Chemical homeostasis & GO:0048878, GO:0055082 & \\
\hline \multicolumn{3}{|l|}{ Molecular function } \\
\hline Ferroxidase activity & GO:0004322 & \multirow{2}{*}{$\begin{array}{l}\text { Glyma01g31300, Glyma07g19060, Glyma03g06420, } \\
\text { Glyma18g43650 }\end{array}$} \\
\hline $\begin{array}{l}\text { Oxidoreductase activity, oxidizing } \\
\text { metal ions }\end{array}$ & GO:0016722, GO:0016724 & \\
\hline Ferric iron binding & GO:0008199 & \\
\hline Isocitrate lyase activity & GO:0004451 & Glyma12g10780, Glyma06g45950 \\
\hline \multicolumn{3}{|l|}{ JL1i vs. JL1ni Down-regulated } \\
\hline \multicolumn{3}{|l|}{ Biological process } \\
\hline Photosynthesis & GO:0015979 & $\begin{array}{l}\text { Glyma09g08630, Glyma09g08260, Glyma10g32080, } \\
\text { Glyma11g35130, Glyma12g34320, Glyma13g36240, } \\
\text { Glyma15g01940 }\end{array}$ \\
\hline Photosynthesis, light harvesting & GO:0009765 & Glyma09G08260, Glyma10g32080, Glyma11g35130 \\
\hline $\begin{array}{l}\text { Oxazole or thiazole metabolic } \\
\text { process }\end{array}$ & GO:0018131, GO:0046484, GO:0052837, GO:0052838 & Glyma06g42080, Glyma20g27990 \\
\hline \multicolumn{3}{|l|}{ Cellular component } \\
\hline Photosystem | \& || & GO:0009521, GO:0009522, GO:0009523, GO:0034357 & $\begin{array}{l}\text { Glyma09g08260, Glyma09g08630, Glyma10g32080, } \\
\text { Glyma11935130, Glyma12g34320, Glyma13g36240, } \\
\text { Glyma15g01940 }\end{array}$ \\
\hline Thylakoid & GO:0044436, GO:0009579 & $\begin{array}{l}\text { Glyma09g08260, Glyma09g08630, Glyma10g32080, } \\
\text { Glyma11935130, Glyma12g34320, Glyma13g36240, } \\
\text { Glyma15g01940 }\end{array}$ \\
\hline \multicolumn{3}{|l|}{ Molecular function } \\
\hline Chlorophyll binding & GO:0016168 & Glyma09g08260, Glyma10g32080, Glyma11g35130 \\
\hline \multicolumn{3}{|l|}{ KF1i vs. KF1ni Up-regulated } \\
\hline \multicolumn{3}{|l|}{ KF1i vs. KF1ni Down-regulated } \\
\hline \multicolumn{3}{|l|}{ Biological process } \\
\hline Photosynthesis & GO:0015979 & $\begin{array}{l}\text { Glyma03g42310, Glyma13g36240, Glyma13g43370, } \\
\text { Glyma15g01940 }\end{array}$ \\
\hline \multicolumn{3}{|l|}{ Cellular component } \\
\hline Photosystem I \& I| & GO:0009521, GO:0034357, GO:0009522, GO:0009538 & $\begin{array}{l}\text { Glyma03g42310, Glyma13g36240, Glyma13g43370, } \\
\text { Glyma15g01940 }\end{array}$ \\
\hline Thylakoid & GO:0044436, GO:0009579 & Glyma03g42310, Glyma13g43370, Glyma15g01940 \\
\hline
\end{tabular}

two methods, the relative expression measurement from qRT-PCR was transformed into fold change by base 2 to match with the RNA-seq fold change value. The 7 selected genes for this comparison included five defense responsive genes i.e. Glyma08g08810, Glyma04g20330, Glyma04g10940, Glyma16g06940 and Glyma06g40710 and two WRKYs. Results of comparison between qRTPCR and RNA-seq showed similar expression patterns indicating they were correlated. Therefore, the RNA-seq data could be used for gene expression profiling of soybean in response to Pm-inoculation. The primer set used for qRT-PCR were listed in Additional file 6: Table S6.

\section{Discussion}

The sequences of soybean genome publicly available at Phytozome (http://www.phytozome.net/soybean) coupled with 
Table 2 SDM defense responsive DEGs with $P$ value $<0.001$ and $\log _{2}$ fold change $>2$ or $<-2$

\begin{tabular}{|c|c|c|c|c|c|c|c|c|c|}
\hline \multirow[t]{2}{*}{ Terms } & \multirow{2}{*}{$\begin{array}{l}\text { Gene } \\
\text { ID(Glyma) }\end{array}$} & \multicolumn{4}{|c|}{ Read counts } & \multicolumn{4}{|c|}{ Log2 fold change } \\
\hline & & KF1ni & KF1i & JL1ni & JL1i & KF1 vs. KF1ni & JL1ni vs. KF1ni & JL1i vs. KF1ni & JL1i vs. JL1ni \\
\hline $\begin{array}{l}\text { Plant hormone signal } \\
\text { transduction }\end{array}$ & 11 & & & & & & & & \\
\hline AUX & 2 & & & & & & & & \\
\hline AUX/IAA & $13 g 18910$ & 115.33 & 968.76 & 213.16 & 2345.50 & 3.07 & 0.89 & 4.35 & 3.46 \\
\hline $\mathrm{GH} 3$ & $02 g 13910$ & 43.49 & 373.79 & 84.81 & 398.11 & 3.10 & 0.96 & 3.19 & 2.23 \\
\hline Brassinosteroid & 1 & & & & & & & & \\
\hline BAK1 & $05 g 24770$ & 108.11 & 1407.49 & 1045.10 & 560.86 & 3.70 & 3.27 & 2.38 & -0.90 \\
\hline Ethylene & 1 & & & & & & & & \\
\hline EBF1 & 04g20330 & 32.24 & 586.80 & 184.34 & 2353.68 & 4.19 & 2.52 & 6.19 & 3.67 \\
\hline Jasmonate & 7 & & & & & & & & \\
\hline \multirow[t]{6}{*}{ JAZ1 } & $01 g 41290$ & 78.44 & 1286.07 & 44.57 & 542.98 & 4.04 & -0.82 & 2.79 & 3.61 \\
\hline & 09g08290 & 150.06 & 7641.92 & 382.12 & 8135.14 & 5.67 & 1.35 & 5.76 & 4.41 \\
\hline & $11 \mathrm{~g} 04130$ & 24.65 & 631.92 & 1.67 & 315.76 & 4.68 & -3.89 & 3.68 & 7.57 \\
\hline & $13 g 17180$ & 56.70 & 2397.55 & 73.33 & 3688.38 & 5.40 & 0.37 & 6.02 & 5.65 \\
\hline & $15 g 19840$ & 265.77 & 7262.01 & 432.97 & $10,025.92$ & 4.78 & 0.70 & 5.24 & 4.53 \\
\hline & $17 g 05540$ & 7.67 & 306.83 & 19.98 & 222.05 & 5.32 & 1.38 & 4.86 & 3.47 \\
\hline JAZ6 & 07g04630 & 646.79 & 2896.85 & 524.87 & 3135.59 & 2.16 & -0.30 & 2.28 & 2.16 \\
\hline Plant/pathogen interaction & 31 & & & & & & & & \\
\hline Oxidation & 8 & & & & & & & & \\
\hline Glyoxylase & 07g03560 & 91.03 & 227.06 & 313.50 & 2652.58 & 1.32 & 1.78 & 4.87 & 3.08 \\
\hline \multirow[t]{2}{*}{ Isocitrate lyase } & $06 g 45950$ & 10.77 & 873.50 & 1262.32 & 9650.25 & 6.34 & 6.87 & 9.81 & 2.93 \\
\hline & $12 \mathrm{~g} 10780$ & 116.44 & 1546.11 & 1259.75 & $14,926.85$ & 3.73 & 3.44 & 7.00 & 3.57 \\
\hline \multirow[t]{2}{*}{ Peroxidase } & $01 \mathrm{~g} 32270$ & 0.00 & 2.33 & 2.50 & 73.06 & $\operatorname{lnf} f^{a}$ & $\operatorname{lnf} f^{a}$ & $\operatorname{lnf}^{a}$ & 4.87 \\
\hline & $09 g 41440$ & 1.55 & 120.58 & 4.75 & 227.35 & 7.04 & 1.62 & 7.20 & 5.58 \\
\hline \multirow[t]{3}{*}{ Peroxidase 62-related } & 08g19180 & 1.03 & 451.44 & 4.23 & 364.86 & 8.77 & 2.04 & 8.47 & 6.43 \\
\hline & $15 g 05810$ & 0.52 & 67.97 & 1.21 & 5.65 & 7.04 & 1.22 & 3.44 & 2.22 \\
\hline & $15 g 05820$ & 74.82 & 3413.46 & 94.09 & 577.25 & 5.51 & 0.33 & 2.95 & 2.62 \\
\hline Calmodulin & 12 & & & & & & & & \\
\hline ACA4 & $03 g 31420$ & 202.96 & 1875.99 & 760.97 & 4474.46 & 3.20 & 1.91 & 4.46 & 2.56 \\
\hline \multirow[t]{2}{*}{$C M L$} & $\log 32190$ & 14.00 & 788.07 & 195.32 & 626.99 & 5.81 & 3.80 & 5.49 & 1.68 \\
\hline & $10 g 00470$ & 6.00 & 270.76 & 94.11 & 1064.77 & 5.50 & 3.97 & 7.47 & 3.50 \\
\hline CML24 & $18 g 04450$ & 346.73 & 1903.08 & 30.25 & 301.65 & 2.46 & -3.52 & -0.20 & 3.32 \\
\hline CML44 & $13 G 09550$ & 30.16 & 736.89 & 159.44 & 1104.95 & 4.61 & 2.40 & 5.20 & 2.80 \\
\hline CML1-related & 05g13900 & 2618.57 & $36,645.18$ & 4992.34 & $30,027.30$ & 3.81 & 0.93 & 3.52 & 2.59 \\
\hline \multirow[t]{2}{*}{ CML-like } & $03 g 39170$ & 19.28 & 853.73 & 214.17 & 4922.86 & 5.47 & 3.47 & 8.00 & 4.52 \\
\hline & 03g39180 & 57.14 & 1443.30 & 31.25 & 8369.36 & 4.66 & -0.87 & 7.20 & 8.06 \\
\hline \multirow[t]{2}{*}{ CML24-like } & 05g33880 & 172.33 & 3470.54 & 438.99 & 3944.01 & 4.33 & 1.35 & 4.52 & 3.17 \\
\hline & 08g05810 & 149.12 & $12,677.75$ & 239.19 & $13,111.02$ & 6.41 & 0.68 & 6.46 & 5.78 \\
\hline CML44-like & $14 g 24810$ & 4.90 & 702.56 & 71.12 & 517.40 & 7.16 & 3.86 & 6.72 & 2.86 \\
\hline CML-dependent & $11 \mathrm{~g} 13740$ & 191.84 & 12.92 & 7.56 & 1.41 & -3.89 & -4.67 & -7.10 & -2.42 \\
\hline PR proteins & 11 & & & & & & & & \\
\hline \multirow[t]{2}{*}{ PR proteins } & 01g31730 & 0.52 & 74.89 & 11.38 & 2174.33 & 7.18 & 4.45 & 12.03 & 7.59 \\
\hline & $03 g 30420$ & 3.22 & 305.06 & 507.98 & 1023.66 & 6.56 & 7.30 & 8.31 & 1.02 \\
\hline
\end{tabular}


Table 2 SDM defense responsive DEGs with $P$ value $<0.001$ and $\log _{2}$ fold change $>2$ or $<-2$ (Continued)

\begin{tabular}{|c|c|c|c|c|c|c|c|c|c|}
\hline \multirow[t]{2}{*}{ Terms } & \multirow{2}{*}{$\begin{array}{l}\text { Gene } \\
\text { ID(Glyma) }\end{array}$} & \multicolumn{4}{|c|}{ Read counts } & \multicolumn{4}{|c|}{ Log2 fold change } \\
\hline & & KF1ni & KF1i & JL1ni & JL1i & KF1 vs. KF1ni & JL1ni vs. KF1ni & JL1i vs. KF1ni & JL1i vs. JL1ni \\
\hline & $06 g 40710$ & 133.49 & 903.17 & 0.90 & 7.30 & 2.76 & -7.22 & -4.19 & 3.01 \\
\hline & $16 g 06940$ & 165.99 & 1996.03 & 834.75 & 2864.73 & 3.59 & 2.33 & 4.11 & 1.78 \\
\hline \multirow[t]{2}{*}{ FLS2 } & 08g08810 & 49.37 & 3483.31 & 19.60 & 613.61 & 6.14 & -1.33 & 3.64 & 4.97 \\
\hline & $08 g 08781$ & 2.19 & 161.18 & 0.00 & 26.67 & 6.20 & 0.00 & 3.61 & $\operatorname{lnf}^{a}$ \\
\hline LOX1 & $13 g 31280$ & 136.67 & 1071.67 & 68.13 & 87.54 & 2.97 & -1.00 & -0.64 & 0.36 \\
\hline RPW8 & $01 \mathrm{~g} 32825$ & 12.50 & 473.23 & 5.45 & 60.99 & 5.24 & -1.20 & 2.29 & 3.49 \\
\hline PEPR1 & $\log 33970$ & 13.48 & 321.96 & 38.23 & 693.94 & 4.58 & 1.50 & 5.69 & 4.19 \\
\hline \multirow[t]{2}{*}{ PEPR1/2 } & $12 \mathrm{~g} 00960$ & 66.44 & 797.47 & 340.30 & 710.24 & 3.59 & 2.36 & 3.42 & 1.06 \\
\hline & $13 g 32630$ & 17.61 & 367.59 & 348.28 & 1724.20 & 4.38 & 4.31 & 6.61 & 2.31 \\
\hline Transcription factors & 10 & & & & & & & & \\
\hline bHLH & 1 & & & & & & & & \\
\hline bHLH103 & $15 g 07010$ & 27.74 & 354.74 & 71.50 & 993.14 & 3.68 & 1.37 & 5.16 & 3.80 \\
\hline MYB & 6 & & & & & & & & \\
\hline \multirow[t]{2}{*}{ MYB-related } & 09g29800 & 216.98 & 1442.26 & 121.66 & 1074.53 & 2.73 & -0.83 & 2.31 & 3.14 \\
\hline & $\log 05560$ & 39.98 & 288.14 & 20.12 & 270.80 & 2.85 & -0.99 & 2.76 & 3.75 \\
\hline MYB32- related & $02 g 41180$ & 36.70 & 93.29 & 72.15 & 685.06 & 1.35 & 0.98 & 4.22 & 3.25 \\
\hline \multirow[t]{2}{*}{ MYB-related TF LHY } & $03 g 42260$ & 183.04 & 981.94 & 69.66 & 598.94 & 2.42 & -1.39 & 1.71 & 3.10 \\
\hline & $19 g 45030$ & 215.40 & 1042.06 & 125.26 & 1205.87 & 2.27 & -0.78 & 2.49 & 3.27 \\
\hline MYB-like & $03 g 34110$ & 15.35 & 189.20 & 68.03 & 911.41 & 3.62 & 2.15 & 5.89 & 3.74 \\
\hline WRKY & 3 & & & & & & & & \\
\hline WRKY4 & $01 \mathrm{~g} 31921$ & 43.63 & 765.05 & 139.06 & 2145.04 & 4.13 & 1.67 & 5.62 & 3.95 \\
\hline WRKY31 & $04 g 06495$ & 4.12 & 145.38 & 103.06 & 341.37 & 5.14 & 4.65 & 6.37 & 1.73 \\
\hline WRKY156 & $17 \mathrm{~g} 04710$ & 7.48 & 154.07 & 138.48 & 52.69 & 4.36 & 4.21 & 2.82 & -1.39 \\
\hline
\end{tabular}

inf $^{\mathrm{a}}$ represents infinitive fold change

Bold data represent the total number of DEGs belong to different terms

availability of next-generation RNA sequencing analysis provided a powerful tool to study the differential expression of genes in soybean plants in response to biotic and abiotic stresses. Kim et al. (2011) reported the first application of RNA-seq for profiling gene expression in soybean in response to pathogen attack. In their study, the transcriptomes of two near isogenic lines (NILs), one resistant and one susceptible to bacterial leaf pustule (Xanthomonas axonopodis pv. glycines), were analyzed 0, 6, and 12 hai and a total of 2761 DEGs, including a set of defense response genes, were identified. In the following years, transcriptomic analyses were reported on Asian soybean rust (Phakopsora pachyrhizi) [11], soybean root and stem rot (Phytophthora sojae) [10] in soybean, and soybean cyst nematode (SCN; Heterodera glycines) in common bean [12]. By which the responsive genes and metabolism pathways associated with disease resistance have been identified. Unfortunately, no such a study in soybean $/ \mathrm{Pm}$ interaction has been reported so far. Here, we provided data demonstrating there were significant transcriptomic variations in
SDM-resistant and susceptible genotypes of soybean in response to Pm-infection.

\section{SDM resistance and inoculation}

In our previous study, we cloned a GmSAGT1 gene from a HR genotype of soybean, Zaofeng5. After $4 \mathrm{~h}$ of SA induction, the transcript level of this gene in the HR genotype was 13 times higher than that in HS genotype Heinong10, demonstrating that higher expression of GmSAGT1 gene was associated with increased SDM resistance [7]. In the current study, we used an additional pair of genotypes i.e. JL1 (HR) and KF1 (HS) as plant materials for conducting transcriptomic analysis. JL1 was bred by the Soybean Institute, Jilin Academy of Agricultural Sciences in 1979. This cultivar was derived from a cross between cultivated soybean (G. max) and wild spices (G. sojae, GD50477). It featured as small grain size, relative narrow and long leaves, early maturity and higher resistance to SDM. These characters might be derived from wild species of soybean [13, 14]. The 
KF1 was a commercial variety in late 1990s with SMV (soybean mosaic virus) resistance that later on turned to be highly susceptible to SDM.

For understanding differential expression of genes in response to $\mathrm{Pm}$ infection, it is important to know the sampling time point corresponding to what stage of pathogen invasion. In this study, 72 hai was used. According to the literature, the 72 hai was corresponding to the stage of hyphae growth and haustoria formation of $P m$ fungi in the soybean plant tissue. Riggle (1974) conducted a detailed histopathological study of $\mathrm{Pm}$ on leaves of soybean cultivars with varying degree of resistance and on a non-host. It indicated that the germination of conidia, germ tube growth, appressorium formation, development of penetration pegs, and hyphal growth for 36 hai were about the same on all categories of plants, including a susceptible, a resistant near isogenic line (NIL), a heterozygous line for resistance, a moderately resistant cultivar and a non-host common bean. During 36 to 120 hai, hyphae grew rapidly on the leaves of susceptible cultivars and the number of haustoria/mm of hyphae was high. However, on the resistant NIL, the hyphae growth was very slow and few haustoria were formed. The author concluded that the resistance appeared to be related to slow rate of hyphal growth and the ability of hyphae to form haustoria. These data suggested that the 72 hai used in the current study was the right time point to represent the early stage of $\mathrm{Pm}$ invasion. The DEGs identified in the HR and HS genotype at this stage might provide insight and expand our knowledge in understanding the molecular basis of SDM resistance.

\section{SDM defense responsive genes}

By comparison of the read counts of the 52 SDMdefense responsive DEGs, it was interesting to find that the expression levels of most DEGs (73\%) in HR noninoculated samples were higher than that in the HS non-inoculated ones (Table 2). It indicated that the basal expression levels of these DEGs might be sufficient for resistance and could be serve as basal defense responsive genes. In contrast, by comparison of the $\log _{2}$ fold change value of inoculated vs non-inoculated samples of $\mathrm{HR}$ and HS, it showed that the susceptible KF1 reacted to $P m$ attack more rapidly and strongly (Table 2 ). This was not surprising since SDM lesions were developed on leaves of HS plant 72 hai, while during this period only few hypersensitive spots showed on leaves of HR plant. Although HS/KF1 responded to fungal attack more quickly, the read counts of DEGs (30/52, 58\%) were still lower than that in HR/JL1 after inoculation (Table 2). It should also note that, after $\mathrm{Pm}$ inoculation, the $\log 2$ fold change value of $83 \%$ DEGs in HR and 96\% DEGs in HS were greater than 2.0 (Table 2), demonstrating additive effects existed following fungal invasion.

Plants have developed two defense mechanisms in its evolution, which are (i) pathogen triggered immunity (PTI) that confers non-host resistance by recognizing a broad range of pathogens with conserved molecular pattern. (ii) effectors-triggered immunity (ETI), in which the pathogen effectors are recognized by specific $R$ genes that encode nucleotide binding site and NBS-LRR domains $[15,16]$. Unfortunately, to the best of our knowledge, the ETI genes specifically resistant to $P m$ have not yet been reported to date in soybean due to both genetic and pathogenic analysis at molecular level are lacking. As such, the gene-for-gene interaction between soybean and $P m$ is unclear so that it is not possible to classify the putative genes detected in this study into PTI and ETI. Therefore, we further analyzed the genes involving in phytohormone signalling pathways and the genes encoding PR proteins as well as TFs based on the existing knowledge and the functional annotation of DEGs in GO and KEGG analysis in the study.

It has been shown that SA, JA and ET play major roles in response to biotic stress as their levels increase with pathogen infection $[17,18]$. Upon pathogen infection, the early signalling events occurred, including increase of intracellular $\mathrm{Ca}^{2+}$ concentration and production of secondary signalling molecules such as ROS. In our GO analysis, 5 of the most enriched DEGs (iron ion homeostasis, metal ion homeostasis, cation homeostasis, ion homeostasis, chemical homeostasis) under the category of biological process were all associated with ion homeostasis and 4 DEGs (ferroxidase activity, oxidoreductase activity, ferric iron binding, isocitrate lyase activity) in the category of molecular function were associated with oxidation as well as ROS production (Table 1). In the analysis of SDM defense responsive genes, 10 DEGs coding for calmodulin proteins and 1 coding for calmodulin-regulated $\mathrm{Ca}^{2+}$ ATPase (ACA4) were identified (Table 2), indicating a wide range of genes participated in the modulation of $\mathrm{Ca}^{2+}$ metabolism.

Studies have shown that $\mathrm{Ca}^{2+}$ functions in concert with other important second messengers like ROS [19]. In the SA signalling pathway, a rapid increase in the rate of ROS production, known as 'the oxidative burst', occurs in response to biotic and abiotic stresses [20]. Higher rate of ROS production results in the induction of PR genes expression which in turn leading to a longlasting and broad-spectrum induced resistance known as systemic acquired resistance (SAR) [18]. Once SA pathway is activated, the expression of downstream PR genes is induced. Results obtained in this study indicated there were four genes encoding PR proteins (Glyma01g31730, Glyma03g30420, Glyma06g40710, Glyma16g06940) that were highly differential expressed (Table 2). 
In soybean, our previous study indicated that after SA induction, the peroxisome-located gene GmSAGT1 and its upstream gene $G m G O X$ coding for glycolate oxidase were highly expressed in soybean resistant variety than that in susceptible ones [7]. The function of GmGOX protein is to convert glycolic acid into glyoxylate and $\mathrm{H}_{2} \mathrm{O}_{2}$ by oxidation [21]. Interestingly, in the current study we have identified seven DEGs coding for oxidative enzymes, including one glyoxylase, two isocitrate lyases and four peroxidases. It is known the isocitrate lyase is a key enzyme in the glyoxylic acid cycle, which catalyzes the lysis of isocitric acid to produce succinic acid and glyoxylic acid. Subsequently, the resultant glyoxylic acid is further converted to serine and $\mathrm{H}_{2} \mathrm{O}_{2}$. These findings have provided evidence that SA in conjunction with ROS appear to be involved in the establishment of systemic defenses and play an essential role in $\mathrm{H}_{2} \mathrm{O}_{2}$ accumulation, $\mathrm{PR}$ gene expression, hypersensitive response and SAR induction [22].

$\mathrm{SA}$ is generally involved in the activation of defense responses against biotrophic and hemi-biotrophic pathogens [23], whereas, JA and ET are responsible for defense against necrotrophic pathogens $[24,25]$. The $P$. manshurica is a biotrophic pathogen and the annotation of the most DEGs identified in this study let us reasonably to suggest SA signalling coupled with ROS is a key prominent pathway in soybean in response to $\mathrm{Pm}$ infection. Based on above results, a schematic diagram was drawn in describing the molecular processes and the involvement of DEGs in soybean host upon Pm-invasion (Fig. 5).

Recent studies have shown that there is sophisticated crosstalk between different hormone-mediated pathways. In a recent review article, Verma et al. (2016) have

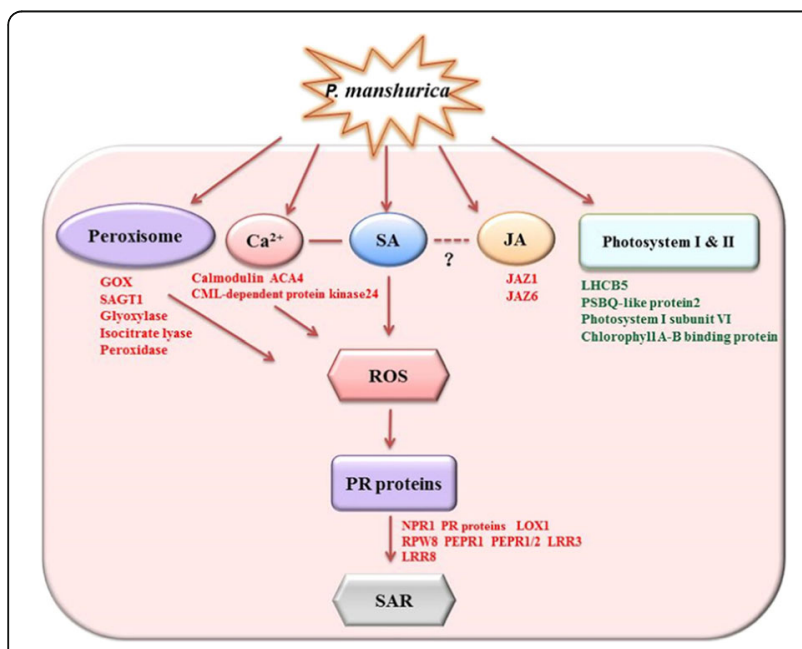

Fig. 5 A schematic diagram of soybean/Pm interaction showing the molecular processes and the involvement of DEGs identified in this study. Note: the DEGs with red color are up-regulated while the DEGs in green are down-regulated emphasized that the defense responses activated in plants in response to different stresses depends on the type of crosstalk (positive or negative) between the hormone signaling pathways rather than solely on the individual contributions of each hormone.

It is commonly viewed that SA and JA regulate biotic stress responses antagonistically [17]. Studies have shown NPR1 to be a key player in the antagonistic crosstalk of SA and JA [26]. The SA-facilitated suppression of JA-responsive genes like LIPOXYGENASE 2 (LOX2) has been reported [27]. Interestingly, an isozyme LOX1 was also detected in the present study, however, it was upregulated after $\mathrm{Pm}$ inoculation. Moreover, it is noteworthy that, in contrast to antagonistic crosstalk between SA and JA, we found they are simultaneously activated since seven JAZ and two FLS2 were highly differential expressed in both HR/JL1 and HS/KF1 after Pm infection. Although most studies prove antagonistic interaction between SA and JA, synergistic interactions have also been observed at low SA-JA concentrations and by simultaneous induction of both defenses [28, 29].

The DEGs involving in ET, BR and AUX signalling pathways were detected in this study. It was reported that JA and ET operate synergistically in regulating defense related genes after pathogen infection [30]. However, in considering JA and ET are responsible for defense against necrotrophic pathogens [30] and auxin is mainly responsible for plant growth and development [18], the roles of these signalling in SDM defense responses require further elucidation. Due to the complexity of crosstalk between different plant hormones and in line with the simultaneous activation of SA and JA observed in this study, it is of great interest to investigate how they crosstalk one another and whether they are synergistically reacted. All of these remain to be addressed.

\section{Transcription factors}

In the study, three types of TFs including one bHLH (the core JA-signalling components MYC2), six MYBs and three WRKYs, which differentially expressed in HR/ JL1i vs. JL1ni and HS/KF1i vs. KF1ni, were identified (Table 2).

MYC2 is a basic helix-loop-helix (bHLH) TF that recognizes the G-box and G-box variants in the promoter of its target genes and regulates different branches of the JA pathway [31-37]. MYB1 gene in tobacco is known to encode a signaling component downstream of SA that may participate in transcriptional activation of PR genes and plant disease resistance [38]. MYBs and WRKYs are large families in plant genome, in soybean there are 252 and 188 members of MYB [39] and WRKY families [40], respectively. 
WRKY proteins, a large family of transcriptional regulators that has to date only been found in plants. Many previous studies have shown WRKYs are involved in the plant response to biotic and abiotic stresses either act as transcriptional activators or repressors [41-43]. In soybean, considerable effort has gone into identifying WRKY TFs and its mediated gene expression in response to pathogen invasion. Bencke-Malato et al. [11] have conducted a detailed study on genome-wide annotation of the soybean WRKY family and functional characterization of genes involved in response to Phakopsora pachyrhizi (Asian soybean rust) infection. In this study, we have identified three WRKYs, i. e. GmWRKY4 (Glyma01g31921), 31 (Glyma04g06495) and 156 (Glyma17g04710) (the name of GmWRKYs are given according to the $\mathrm{Yu}$ et al., [40]), that are highly differential expressed following $P m$ inoculation. In particular, their expression level has increased 4.13- to 5.14-fold in HS/KF1, while it has increased 1.39- to 3.95fold in HR/JL1. To date, the roles of WRKYs in soybean immune response to different pathogen attack and how WRKYs react with their target genes or crosstalk to the genes involving in the SA-, JA- and other plant hormone signalling remain largely unknown.

\section{Conclusion}

Comparative transcriptomic analysis of $\mathrm{HR}$ and $\mathrm{HS}$ genotype with and without inoculation allowed us to explore defense responsive genes to $P$. manshurica infection. Totally, 52 DEGs were identified in the study, which participated in the SA/ROS and JA signalling pathway resulting in the calcium modulation, oxidative burst and PR protein production. Of particular interest are the DEGs coding for glyoxylase, isocitrate lyase and an array of peroxidase. The PR proteins and TFs are also worth to be further investigated and characterized to identify candidate genes resistant to soybean downy mildew disease. As plant defense response is a very complex system, which needs further comprehensive analysis step by step.

\section{Methods}

\section{Plant materials and $P m$-infection}

In our previous study [7], 15 soybean genotypes were classified into five categories: HR (highly resistant), R (resistant), MR (moderate resistant), S (susceptible) and HS (highly susceptible) by using in vitro leaf $/ P m$ inoculation or by field observation. In the current study, we have used JL1 (HR) and KF1 (HS) as plant materials for conducting transcriptomic analysis.

To prepare source of $\mathrm{Pm}$, infected leaves (mixture of different races) were collected from soybean field at Shenyang Agricultural University. Collected leaves were placed on moisture paper in a Petri dish and maintained at $20{ }^{\circ} \mathrm{C}$ overnight for proliferation of conidia. Leaves were then washed with autoclaved double distilled water to collect conidia followed by dilution to a final concentration of $5 \times 10^{5}$ conidia $/ \mathrm{ml}$.

Back side of the top third expanded leaves of $40 \mathrm{~d}$-old seedlings were inoculated using brush pen. Leaves of JL1 and KF1 brushed with distilled water in the same manner without pathogen were used as a control. Plants were then placed in a growth chamber under conditions of $20 / 24{ }^{\circ} \mathrm{C}, 14 \mathrm{~h}$ dark/10 h light cycle and relative humidity $\geq 95 \%$.

\section{Sample preparation and RNA sequencing}

Total RNA was extracted from leaves 72 hai using RNAprep Pure Plant Kit (Tiangene Co., China) following the manufacturer's instructions. To prepare cDNA samples for transcriptome sequencing, the quantity of total RNA was assessed with Nanodrop spectrophotometer (Thermo Fisher Scientific Inc., USA). The RNA quality and integrity was evaluated on the Agilent Bioanalyzer 2100 system (Agilent Technologies, USA) and then processed with NEBNext Ultra RNA Library Prep Kit. The cDNA fragments of $\sim 150 \mathrm{bp}$ in length were selected and further enriched with PCR amplification to generate cDNA libraries. Four cDNA libraries derived from JL1ni, JL1i, KF1ni and KF1i, each with two biological replicates, were used for Illumina sequencing. RNA-sequencing was conducted by Allwegene Co., China using equipment of HiSeqTM2000/ MiSeq, which were technically repeated three times.

\section{Reads filtering, alignment and de novo assembly}

Before assembly, the raw reads were first filtered to gain high quality clean reads by removing low quality reads as well as reads containing adaptor or unknown nucleotides larger than $10 \%$. Clean reads were de novo assembled into transcripts based on the soybean reference genome sequence. For removing redundancy, when a component contained more than one assembled transcript, only the longest one was preserved. The clean reads from each RNA-seq library were aligned with soybean genome (Glycine $\max$ Wm82.a2.v1) as a reference (http://www.phytozome.net/soybean) using TopHat software (http://tophat.cbcb.umd.edu). Resulted read counts for each matched gene were normalized with RPKM (reads per kilo bases per million reads) to measure its expression level. For gene annotation, the matched genes were searched by BLAST against the databases of Phytozome and NCBI (www.ncbi.nlm.nih.gov/).

\section{Differential expression of genes in response to $P m$ infection}

Genes differentially expressed in JL1i vs. JL1ni and KF1i vs. KF1ni with $P$-value $<0.001$ were selected and the difference of expression level was $\log _{2}$-transformed and 
filtered at a level of 2-fold or greater. These genes were considered as DEGs. DEGs in inoculated and noninoculated leaves of HR and HS genotype were represented as Venn diagrams.

Annotation of the up- and down-regulated genes was carried out based on the identification of conserved PFAM domains (pfam.xfam.org). PFAM domains were converted into GO-identities by mapping to GO (http:// www.geneontology.org).

DEGs were further clustered according to the expression patterns using $\mathrm{H}$-cluster method. The significantly enriched GO terms involving in biological process, cellular component and molecular function in response to soybean $/ P m$ interaction were identified. In addition, the DEGs were mapped to soybean KEGG database using the DEGseq package [44] to search the genes involving in different KEGG pathways. To identify genes in soybean immune responses to $P m$ infection, the up- and down-regulated DEGs and TFs were analyzed based on annotation of their function.

\section{Validation of RNA-seq data by qRT-PCR}

Seven DEGs were randomly selected for qRT-PCR analysis for validation of RNA-seq data. In brief, $1.0 \mu \mathrm{g}$ of total RNA prepared from each sample was first reverse transcribed into its cDNA with oligo(dT) 18 through MMLV Reverse Transcriptase (TaKaRa, Japan). The qPCR was then performed on an ABI 7500 Real-Time System (Applied Biosystems, USA) with a $20 \mu \mathrm{l}$ reaction system. In which it contained $2 \mu \mathrm{l}$ of cDNA, $0.8 \mu \mathrm{l}$ of each primer, $6 \mu \mathrm{l}$ of sterile water, $10 \mu \mathrm{l}$ of SYBR premix TagII (TaKaRa, Japan), and $0.4 \mu$ l of Rox Reference DyeII (TaKaRa, Japan). The conditions for PCR amplification were: $15 \mathrm{~s}$ denaturation at $95^{\circ} \mathrm{C}$ followed by 40 cycles at $95{ }^{\circ} \mathrm{C}$ for $5 \mathrm{~s}, 60{ }^{\circ} \mathrm{C}$ for $34 \mathrm{~s}$, and $72{ }^{\circ} \mathrm{C}$ for $10 \mathrm{~s}$. Primers used for qRT-PCR were designed by DNAMAN software and listed as Additional file 6: Table S6.

Soybean Actin gene (Glyma 08g19420) was used as an internal control. Samples were analyzed based on the stable expression level of the reference gene according to the method described by Livak \& Schmittgen [45]. Three independent replicates were performed for each sample. The data of mean and standard deviation were calculated and statistically analyzed by Student's T-test.

\section{Additional files}

Additional file 1: Table S1. Overview of the sequencing reads. Note: a adaptors and low-quality reads were excluded. b Q20: The percentage of bases with quality value larger than 20. c Q30: The percentage of bases with quality value larger than 30. JL1: HR genotype. KF1: HS genotype. i: inoculated. ni: non-inoculated. (DOCX 18 kb)

Additional file 2: Table S2. Statistics of clean reads mapped to soybean reference genome. Note: JL1: HR genotype. KF1: HS genotype. i: inoculated. ni: non-inoculated. (DOCX $17 \mathrm{~kb}$ )
Additional file 3: Table S3. GO terms identified in soybean HR (JL1i vs. JL1ni) and HS (KF1i vs. KF1ni). Note: A total of 2581 genes were mapped and categorized to GO terms. Data show the number of genes that were up- or down-regulated within the categories of biological process, cellular component and molecular function. The * represents DEGs with significant difference at a level of corrected $P$ value $<0.05$. (DOCX $23 \mathrm{~kb}$ )

Additional file 4: Table S4. KEGG classification of the differentially expressed genes in HR/JL1. Note: Up- and down-regulated NCBI-Gene IDs are non-underlined and underlined, respectively. (DOCX $19 \mathrm{~kb}$ )

Additional file 5: Table S5. KEGG classification of the differentially expressed genes in the HS/KF1. Note: Up- and down-regulated NCBI-Gene IDs are non-underlined and underlined, respectively. (DOCX $19 \mathrm{~kb}$ )

Additional file 6: Table S6. Primers used for qRT-PCR amplification of seven DEGs. Note: F: forward primers; R: reverse primers. (DOCX 17 kb)

\section{Abbreviations}

ACA4: Calmodulin-regulated Ca ${ }^{2+}$-ATPase; AUX: Auxin; bHLH: Basic helixloop-helix; BR: Brassinosteroid; DEGs: Differentially expressed genes; EFB1/ 2: EIN3-binding F-box protein1-like; ETI: Effectors-triggered immunity; FLS2: Flagellin sensing 2; GmSAGT: Serine/alanine glyoxylate amino transferase; GO: Gene ontology; GOX: Glycolate oxidase; HR: Highly resistant; HS: Highly susceptible; IAA: Indole-3-acetic acid; JA: Jasmonic acid; JAZ: JASMONATE ZIM-motif; KEGG: Kyoto encyclopedia of genes and genomes; LOX: Lipoxygenase; LRR: Leucine-rich repeat; NBS-LRR: Nucleotide binding site-leucine rich repeat; NIL: Near isogenic line; Pm: Peronospora manshurica; PR protein: Pathogenesis-related protein; PTI: Pathogen triggered immunity; qRT-PCR: Real-time quantitative PCR; ROS: Reactive oxygen species; SA: Salicylic acid; SAR: Systemic acquired resistance; SDM: Soybean downy mildew; TF: Transcription factor

\section{Acknowledgements}

We sincerely thank Prof. Lijuan Qiu in the National Germplasm Bank, China for kindly providing us seeds of soybean varieties.

\section{Funding}

RNA-sequencing and other experiments were supported by 'The development of new varieties of animals and crops' under the National High Priority R\&D Program (2016ZX08009003-005-006); The Agricultural Science and Technology Innovation Program were used for data analysis and writing the manuscript.

\section{Availability of data and materials}

The transcriptomic analysis of the resistant and susceptible soybean plants in response to fungal pathogen Peronospora manshurica was conducted in this work and the raw data of RNA-seq have been deposited in the NCBI Sequence Read Archive (http://trace.ncbi.nlm.nih.gov/Traces/sra/sra.cgi) and the access code is PRJNA436627.

\section{Authors' contributions}

Experimental design and supervision, YHL and YHW; Investigation, HD, SFS, CZ, SHZ, ML, JT, YY, LPL and XJW; Manuscript writing and revision, SRJ, YHL and $\mathrm{ML}$; Funding acquisition, YHL. All authors read and approved the final manuscript.

\section{Ethics approval and consent to participate}

The plant materials in this research were all provided by the National Germplasm Bank of China. These materials are widely used in laboratory studies and agricultural production. No permits are required for the collection of plant samples. All the experiments were conducted in accordance with China's legislation and the regulations of Chinese Academy of Agricultural Sciences. This article did not contain any studies with human participants or animals, and did not involve any endangered or protected species.

\section{Competing interests}

The authors declare that they have no competing interests.

\section{Publisher's Note}

Springer Nature remains neutral with regard to jurisdictional claims in published maps and institutional affiliations. 


\section{Author details}

'Biotechnology Research Institute, Chinese Academy of Agricultural Sciences, Beijing, China. ${ }^{2}$ College of Plant Protection, Shenyang Agricultural University, Shenyang, China. ${ }^{3}$ School of Biotechnology, East China University of Science and Technology, Shanghai, China.

Received: 7 August 2017 Accepted: 30 April 2018

Published online: 18 May 2018

\section{References}

1. Dunleavy JM. Yield reduction in soybean caused by downy mildew. Plant Dis. 1987;71:1112-4.

2. Lim SM. Inheritance of resistance to Peronospora manshurica race 2 and race 33 in soybean. Phytopathology. 1989;79:877-9.

3. Silva OC, et al. Potassium phosphite for control of downy mildew of soybean. Crop Prot. 2011;30:598-604.

4. Wang JJ, Liu $\sqcup$. The epidemic and control measures of soybean downy mildew in Heilongjiang province of China. Soybean Sc Technol. 2009;1:6.

5. Riggle $\mathrm{JH}$. Histopathology of Peronospora manshurica on soybean cultivars of varying degrees of resistance and on a nonhost. Retrospective theses and dissertations. 1974. P.6362.

6. Fawke S, Doumane, Schornack S. Oomycete interactions with plants: infection strategies and resistance principles. Microbiol Mol Biol Rev. 2015; 79:263-80.

7. Sun WL, Yin C, Li Y, Li M, Zhang HG, Shi SF, et al. Cloning and characterization of a GmSAGT1 gene whose transcriptional expression is associated with the level of resistance to soybean downy mildew. Plant Mol Biol Report. 2015;33:1232-44.

8. Kim KH, Kang YJ, Kim DH, Yoon MY, Moon JK, Kim MY, et al. RNASeq analysis of a soybean near-isogenic line carrying bacterial leaf pustuleresistant and -susceptible alleles. DNA Res. 2011;18:483-97.

9. Kim KH, Kang YJ, Shim S, Seo MJ, Baek SB, Lee, et al. Genome-wide RNA-seq analysis of differentially expressed transcription factor genes against bacterial leaf pustule in soybean. Plant Breed Biotech. 2015;3:197-207.

10. Lin F, Zhao MX, Baumann DD, Ping JQ, Sun LJ, Liu YF, et al. Molecular response to the pathogen Phytophthora sojae among ten soybean near isogenic lines revealed by comparative transcriptomics. BMC Genomics. 2014; https://doi.org/10.1186/1471-2164-15-18.

11. Bencke-Malato M, Cabreira C, Wiebke-Strohm B, Bücker-Neto L, Mancini E, Osorio MB, et al. Genome-wide annotation of the soybean WRKY family and functional characterization of gene involved in response to Phakopsora pachyrhizi infection. BMC Plant Biol. 2014; https://doi.org/10.1186/s12870-014-0236-0.

12. Jain S, Chittem K, Brueggeman R, Osorno JM, Richards J, Nelson BD. Comparative transcriptome analysis of resistant and susceptible common bean genotypes in response to soybean cyst nematode infection. PLoS One. 2016:11:e0159338.

13. Zheng HY, Yang GY, Han CF, Sun YL. Breeding a new soybean variety Jilinxiaoli 1 by hybridation of Glycine sojia. and Glycine max. J Jilin Agric Sci. 1991:3:9-11. (in Chinese)

14. Zheng HY, Yang GY, Han CF, Sun YL. Jilinxiaoli 1-a new soybean exporting variety. China Seeds. 1992;1:46. (in Chinese)

15. Jones JDG, Dangl JL. The plant immune system. Nature. 2006:444:323-9.

16. Dodds PN, Rathjen JP. Plant immunity: towards an integrated view of plantpathogen interactions. Nat Rev Genet. 2010;11:539-48.

17. Bari R, Jones JD. Role of plant hormones in plant defence responses. Plant Mol Biol. 2009:69:473-88.

18. Verma V, Ravindran P, Kumar PP. Plant hormone-mediated regulation of stress responses. BMC Plant Biol. 2016; https://doi.org/10.1186/s12870-016-0771-y.

19. Görlach A, Bertram K, Hudecova S, Krizanova O. Calcium and ROS: a mutual interplay. Redox Biol. 2015; https://doi.org/10.1016/j.redox.2015.08.010.

20. Miller G, Shulaev V, Mittler R. Reactive oxygen signaling and abiotic stress. Plant Physiol. 2008;133:481-9.

21. Tolbert NE. Photorespiration. New York: The biochemistry of plants: Academic Press; 1980. p. 488-525

22. Kanzaki H, Saitoh H, Ito A, Fujisawa S, Kamoun S, Katou S, et al. Cytosolic HSP90 and HSP70 are essential components of INF1-mediated hypersensitive response and non-host resistance to Pseudomonas cichorii in Nicotiana benthamiana. Mol Plant Pathol. 2003;4:383-91.

23. Loake G, Grant M. Salicylic acid in plant defence-the players and protagonists. Curr Opin Plant Biol. 2007;10:466-72.
24. Wasternack C, Hause B. Jasmonates: biosynthesis, perception, signal transduction and action in plant stress response, growth and development. An update to the 2007 review in annals of botany. Ann Bot. 2013;111:1021-58.

25. Gamalero E, Glick BR. Ethylene and abiotic stress tolerance in plants. In: Environmental adaptations and stress tolerance of plants in the era of climate change. New York: Springer; 2012. p. 395-412.

26. Wu Y, Zhang D, Chu JY, Boyle P, Wang Y, Brindle ID, Luca VD, Després C. The Arabidopsis NPR1 protein is a receptor for the plant defense hormone salicylic acid. Cell Rep. 2012;1:639-47.

27. Spoel SH, Koornneef A, Claessens SM, Korzelius JP, Van PJ, Mueller MJ, et al. NPR1 modulates cross-talk between salicylate- and jasmonate-dependent defense pathways through a novel function i n the cytosol. Plant Cell. 2003; 15:760-70.

28. Li J, Brader G, Palva ET. The WRKY70 transcription factor: a node of convergence for jasmonate-mediated and salicylate-mediated signals in plant defense. Plant Cell. 2004;16:319-31.

29. Petersen $M$, Brodersen $P$, Naested $H$, Andreasson $E$, Lindhart $U$, Johansen $B$, et al. Arabidopsis map kinase 4 negatively regulates systemic acquired resistance. Cell. 2000;103:1111-20.

30. Zhu Z, An F, Feng Y, Li P, Xue L, Mu A, et al. Derepression of ethylenestabilized transcription factors (EIN3/EIL1) mediates jasmonate and ethylene signaling synergy in Arabidopsis. Proc Natl Acad Sci U S A. 2011;108:12539-44.

31. Busk PK, Pages M. Regulation of abscisic acid-induced transcription. Plant Mol Biol. 1998:37:425-35.

32. Fujita M, Fujita Y, Maruyama K, Seki M, Hiratsu K, Ohme-Takagi M, et al. A dehydration-induced NAC protein, RD26, is involved in a novel ABAdependent stress-signaling pathway. Plant J. 2004;39:863-76.

33. Fujita $Y$, Fujita $M$, Satoh $R$, Maruyama $K$, Parvez MM, Seki $M$, et al. AREB1 is a transcription activator of novel ABRE-dependent ABA signaling that enhances drought stress tolerance in Arabidopsis. Plant Cell. 2005;17:3470-88.

34. Kagaya Y, Hobo T, Murata M, Ban A, Hattori T. Abscisic acid-induced transcription is mediated by phosphorylation of an abscisic acid response element binding factor, TRAB1. Plant Cell. 2002:14:3177-89.

35. Narusaka $Y$, Nakashima K, Shinwari ZK, Sakuma Y, Furihata T, Abe H, et al. Interaction between two cis-acting elements, ABRE and DRE, in ABAdependent expression of Arabidopsis rd29A gene in response to dehydration and high-salinity stresses. Plant J. 2003:34:137-48.

36. Navarro L, Bari R, Achard P, Lison P, Nemri A. Harberd, et al. DELLAs control plant immune responses by modulating the balance of jasmonic acid and salicylic acid signaling. Curr Biol. 2008;18:650-5.

37. Niu Y, Figueroa P, Browse J. Characterization of JAZ-interacting bHLH transcription factors that regulate jasmonate responses in Arabidopsis. J Exp Bot. 2011;62:2143-54.

38. Yang Y, Klessig DF. Isolation and characterization of a tobacco mosaic virusinducible myb oncogene homolog from tobacco. Proc Natl Acad Sci U S A. 1996:93:14972-7.

39. Du H, Yang SS, Liang Z, Feng BR, Liu L, Huang YB, Tang YX. Genome-wide analysis of the WYB transccirption factor superfamily in soybean. BMC Plant Biol. 2012; https://doi.org/10.1186/1471-2229-12-106.

40. Yu YC, Wang N, Hu RB, Xiang FN. Genome-wide identification of soybean WRKY transcription factors in response to salt stress. Springer Plus. 2016; https://doi.org/10.1186/s40064-016-2647-X.

41. Bakshi M, Oelmuller R. WRKY transcription factors: Jack of many trades in plants. Plant Signal Behav. 2014;9:e27700.

42. Eulgem T, Rushton PJ, Robatzek S, Somssich IE. The WRKY superfamily of plant transcription factors. Trends Plant Sci. 2000;5:199-206.

43. Jiang JJ, Ma SH, Ye NH, Jiang M, Cao HS, Zhang JH. WRKY transcription factors in plant responses to stresses. J Integrative Plant Biol. 2017;59:86-101.

44. Wang LK, Feng ZX, Wang X, Wang XW, Zhang XG. DEGseq: an R package for identifying differentially expressed genes from RNA-seq data. Bioinformatics. 2010;26:136-8.

45. Livak KJ, Schmittgen TD. Analysis of relative gene expression data using real-time quantitative $P C R$ and the 2 (-Delta Delta $C(T)$ ) method. Methods. 2001;25:402-8. 